\title{
Neurogenic component of different models of acute inflammation in the rat knee joint
}

\author{
Francis Y Lam, William R Ferrell
}

\begin{abstract}
This study was performed to investigate whether different models of acute joint inflammation showed a neurogenic component and to establish whether this is mediated through sensory afferent or sympathetic efferent nerve fibres. Intra-articular injection of $2 \%$ carrageenan, $20 \mu \mathrm{g}$ substance $\mathrm{P}, 1 \%$ formalin, and $2 \%$ urate all produced an inflammatory response. Prior surgical denervation of the joint significantly inhibited this response in the carrageenan and formalin models, but not the others. Pretreatment of the joint with $1 \%$ capsaicin (about one week previously) significantly reduced the inflammatory response in all models except formalin. In animals pretreated long term with reserpine (to deplete sympathetic nerve endings of their neurotransmitters) significant reductions occurred in the inflammatory responses to substance $P$ and urate. Intraarticular injection of compound $48 / 80$ produced a marked inflammatory response, which was only significantly reduced by capsaicin pretreatment. These results suggest that both the formalin and carrageenan models of inflammation depend to some extent on the integrity of the sensory innervation of the joint, and thus have a neurogenically mediated component to the inflammatory process they generate. In these models there seems to be little contribution from sympathetic efferent fibres. Each model of inflammation showed a different pattern of response to the pretreatments, suggesting that the mediators of the inflammatory process may differ in each case.
\end{abstract}

Involvement of the nervous system in inflammatory responses has been recognised for almost a century and is based on the observation that antidromic electrical stimulation of dorsal roots or peripheral sensory nerves results in vasodilatation $^{1}$ and increased vascular permeability ${ }^{2}$ in the territory innervated. Lewis, ${ }^{3-5}$ in his classic experiments, first implicated the release of substances from the peripheral terminals of nociceptive afferents in the production of cutaneous wheal and flare responses. Since then, evidence has accumulated in support of the notion that the 'axon reflex' is mediated by unmyelinated afferent fibres. Neurogenic vasodilatation and plasma extravasation have been shown to occur during antidromic nerve stimulation at $C$ fibre strength in both the skin ${ }^{2} 6$ and the joint. ${ }^{78}$ Direct activation of $\mathrm{C}$ polymodal nociceptive afferents in skin is also effective in eliciting an inflammatory response. ${ }^{9}$ There is increasing evidence indicating that substance $P$ contained in sensory $\mathrm{C}$ fibres plays an important part in what has come to be termed 'neurogenic inflammation' for both the $\operatorname{skin}^{2}{ }^{10-12}$ and the

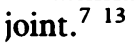

There is another body of thought which suggests that the sympathetic nervous system may contribute to neurogenic inflammation. Evidence supporting this idea arises from the finding that vascular permeability is increased after sympathetic nerve stimulation ${ }^{14}$ and that the baseline plasma extravasation in the knee joint of cats was substantially reduced after lumbosacral sympathectomy. ${ }^{15} 16$ Chemical sympathectomy with 6-hydroxydopamine, ${ }^{17}$ reserpine, ${ }^{18}$ or $\alpha$-methyl- $p$-tyrosine or guanethidine $^{19}$ has been shown to inhibit potently plasma extravasation evoked by intradermal injection of acetic acid, compound $48 / 80$, polymixin B, histamine, and 5-hydroxytryptamine, as well as that produced by thermal injury. Levine et al have also shown that chemical sympathectomy reduces swelling and joint injury in rats with adjuvant arthritis. ${ }^{20}$

The question of whether sensory nerve fibres contribute to the inflammatory process in acute models of joint inflammation has only recently been considered. Carrageenan induced inflammation in the rat knee has been shown to have a significant neurogenic component. ${ }^{21}$ Substance $\mathbf{P}$ has also been shown to be effective in evoking inflammatory processes in the rat knee joint. ${ }^{22}$ It is not known, however, whether the neurogenic component of these inflammatory responses is mediated by the unmyelinated $\mathrm{C}$ fibres or the sympathetic nerves supplying the rat knee, or both. This study is an attempt to identify the types of nerve fibres and the importance of their contributions to the acute models of inflammation produced by carrageenan, substance $P$, formalin, and uric acid. In addition, the effects of the mast cell degranulator, compound $48 / 80$, on plasma extravasation in the rat knee was also investigated.

\section{Materials and methods}

Experiments were performed on male Wistar rats $(\sim 300 \mathrm{~g})$ anaesthetised with a mixture of urethane $(1.13 \mathrm{~g} / \mathrm{kg})$ and diazepam $(2.5 \mathrm{mg} / \mathrm{kg})$. Evans blue (Sigma; $75 \mathrm{mg} / \mathrm{kg}$ ) was injected into the external jugular vein. One knee was injected with $0.2 \mathrm{ml}$ of $0.9 \%$ saline or vehicle to serve as a control while $0.2 \mathrm{ml}$ of either $20 \mu \mathrm{g}$ substance $P$, or $1 \%$ formalin, or $2 \mathrm{mg}$ compound $48 / 80$ was injected into the other knee. At the end of a four hour period the animal was injected with 
Euthatal (133 mg/kg) and exsanguinated. The joint capsule from the anterior and posterior regions of the knee was dissected free and analysed for Evans blue content using a modified technique, ${ }^{23}$ details of which have been reported previously. ${ }^{21}$ As the amount of tissue obtained from one rat is small, in any one experiment the joint capsules from the knees of five rats were used and Evans blue extracted from this pooled sample. The Evans blue values presented throughout represent the difference between the values obtained from knees injected with inflammatory agent and those obtained from saline or vehicle injected (control) knees. The latter samples were necessary to control for the effect of fluid within the synovial cavity and the trauma associated with penetration of the joint capsule.

The procedures for investigating the effects of $2 \%$ carrageenan and $2 \%$ uric acid were essentially the same as above, except that the drugs were allowed to remain in the knee joint for 24 hours and Evans blue injected into the animal four hours before the end of the experiments. Carrageenan and uric acid were found to require a longer period in the synovial cavity (24 hours) to evoke comparable inflammatory responses.

In one group of rats $0.2 \mathrm{ml}$ of $1 \%$ capsaicin $(0.02 \mathrm{~g}$ capsaicin (Sigma) dissolved in $0.1 \mathrm{ml}$ absolute alcohol, $0.1 \mathrm{ml}$ Cremophor (Sigma), and $1.8 \mathrm{ml} 0.9 \%$ saline) was injected into the synovial cavity of one knee under general anaesthesia with Hypnorm $(0.1 \mathrm{mg} / \mathrm{kg})$ and diazepam $(0.5 \mathrm{mg} / \mathrm{kg})$. The rats were allowed to recover and after 9-14 days their response to intra-articular injection of the inflammatory agents into the capsaicin pretreated knee was tested.

In another group of rats the nerves supplying the knee joint were transected unilaterally under general anaesthesia (Hypnorm $0.1 \mathrm{mg} / \mathrm{kg}$; diazepam $0.5 \mathrm{mg} / \mathrm{kg}$ ) and the animals then allowed to recover. After 11-14 days these animals were assessed for their response to the inflammatory agents injected into the denervated knee.

In a further group of rats reserpine $(1 \mathrm{mg} / \mathrm{kg})$ ( $5 \mathrm{mg}$ reserpine dissolved in $1 \mathrm{ml}$ absolute alcohol and $4 \mathrm{ml}(20 \%)$ ascorbic acid) was given intraperitoneally for three successive days. On the fourth day the response of these animals to the inflammatory agents was assessed. In addition, the articular blood flows of six reserpine treated rats in response to electrical stimulation were measured individually. These experiments were performed by laser Doppler flowmetry (Moor Instruments MBF3) on rats under general anaesthesia with a mixture of sodium pentobarbitone $(14 \mathrm{mg} / \mathrm{kg})$, urethane $(142 \mathrm{mg} / \mathrm{kg})$, and diazepam $(0.32 \mathrm{mg} / \mathrm{kg})$. The procedure essentially involved electrical stimulation of the nerve supply to the anteromedial aspect of the knee while monitoring articular blood flow by a laser probe placed on the surface, but not in contact with the anterior portion of the knee joint. A frequency-response curve was obtained by monitoring the changes in the articular blood flow with different frequencies of stimulation $(1-50 \mathrm{~Hz})$. The effectiveness of reserpine treatment in depleting sympathetic neurotransmitters of the articular nerves can be deduced by comparing the frequency-response curve of reserpine treated rats with that of normal rats.

In a final group of rats the effects of the substance $P$ antagonist [D-Pro ${ }^{4}, D-\operatorname{Trp}^{7,9,10}$ ] $\mathrm{SP}_{4-11}$ on the compound $48 / 80$ inflammatory response were investigated by intra-articular injection of $100 \mu \mathrm{g}$ (in $0.1 \mathrm{ml}$ volume) of the antagonist 15 minutes before injection of compound $48 / 80$ into the rat knee.

For each experimental procedure (with the exception of the blood flow experiments, which were performed on individual rats) three to six groups of five rats were used. Data are presented as the mean difference (SEM) in Evans blue content between the control and test knee in each group. Differences were considered significant if the $p$ values were $5 \%$ or less (unpaired $t$ test).

\section{Results}

In the present experiments it was found that intra-articular injection of substance $\mathbf{P}$ (four hours), carrageenan (24 hours), formalin (four hours), and uric acid (twenty four hours) were all effective in producing marked plasma extravasation into the joint capsule. Intra-articular
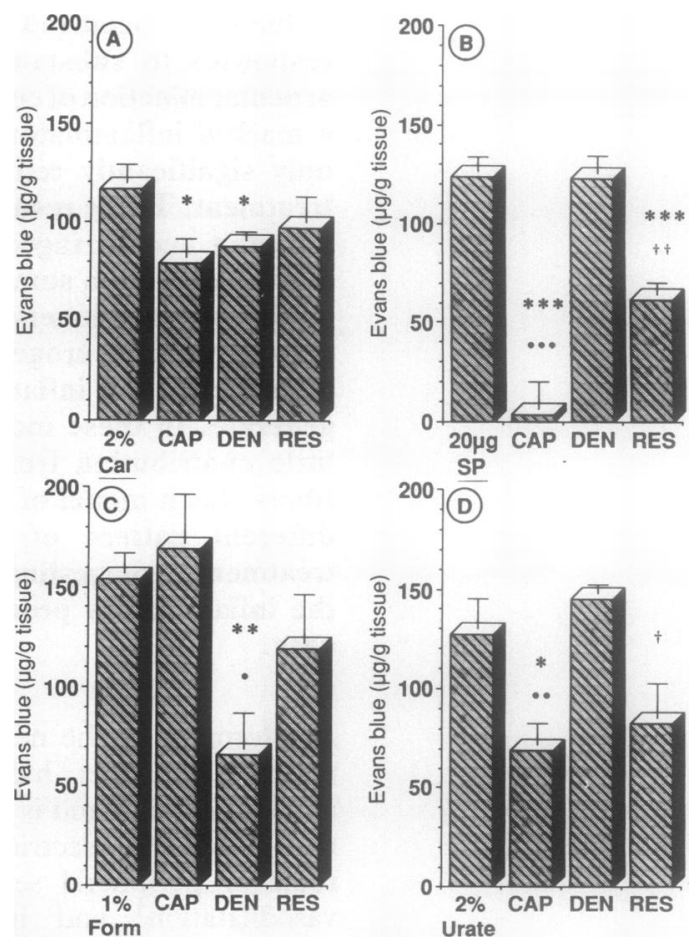

Figure 1 Effect of intra-articular injection of different inflammatory agents on Evans blue content of the joint capsule. Effect of (A) $2 \%$ carrageenan (Car); (B) $20 \mu \mathrm{g}$ substance $P(S P) ;(C) 1 \%$ formalin (Form); (D) $2 \%$ urate. In each histogram the first value gives the control response to injection of these agents alone. The effect of various pretreatments on these responses is given by the adjacent values. $C A P=$ intra-articular injection of $1 \%$ capsaicin about one week previously; $D E N=$ surgical transection of the nerve supply to the knee joint 10 days previously; $R E S=$ reserpine pretreatment. ${ }^{*} p<0.05,{ }^{* *} p<0.01$, ${ }^{* *} p<0.001$ : differs significantly from control. $\bullet p<0.05, \bullet p<0.01, \bullet \bullet \bullet p<0.001$ : capsaicin treatment differs significantly from denervation treatment. $t p<0.05$, H $p<0.01$ : reserpine treatment differs significantly from denervation treatment. 
injection of $1 \%$ capsaicin 9-14 days earlier resulted in a significant reduction $(36 \%)$ of the carrageenan induced inflammatory response. Surgical denervation also produced a significant $25 \%$ reduction in this model, but chemical sympathectomy failed to produce a significant reduction of the inflammatory response (fig 1A).

Substance $\mathbf{P}$ induced inflammation was almost completely abolished (97\% reduction) after capsaicin pretreatment, whereas denervation of the rat knee did not alter the response to substance $P$. Chemical sympathectomy with reserpine, on the other hand, produced a significant $50 \%$ reduction of substance $P$ induced inflammation (fig 1B).

Neither capsaicin pretreatment nor chemical sympathectomy by reserpine produced any significant effects on the formalin induced inflammation, but denervation of the rat knee markedly reduced $(57 \%)$ the formalin response (fig 1C). With uric acid induced inflammation, capsaicin pretreatment produced a significant $46 \%$ reduction, whereas knee joint denervation failed to attenuate the uric acid response significantly (fig 1D). Reserpine pretreatment did produce a reduction in the inflammatory response, but this was not significant compared with the response to uric acid alone, but just reached significance compared with the effect of uric acid in the denervated knee.

Compound 48/80 when injected into the rat knee elicited a very potent inflammatory action, which was not affected by substance $P$ antagonist pretreatment, reserpine pretreatment, or denervation of the rat knee. Inflammation induced by compound 48/80, however, was significantly reduced (26\%) after capsaicin treatment (fig 2).

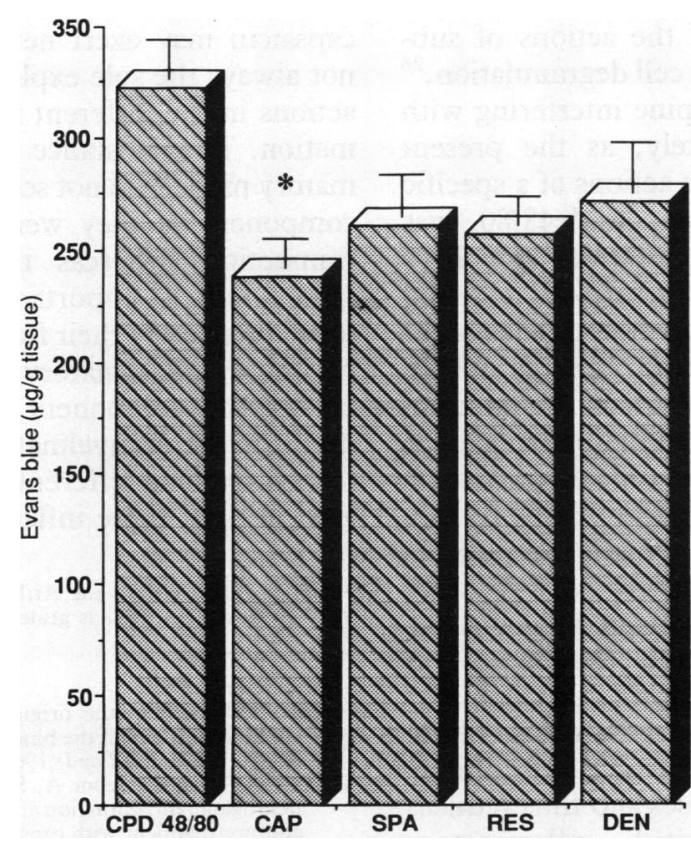

Figure 2 Evans blue content of the rat knee joint capsule (test-control response) four hours after intra-articular administration of $2 \mathrm{mg}$ compound (CPD) 48/80. Although pretreatment by surgical denervation $(D E N)$, reserpine $(R E S)$, and prior intra-articular injection of $100 \mu \mathrm{g}$ of $[\mathrm{D}-$ Pro $\left.^{4}, D-T r p^{7,9,10}\right] S P_{4-11}$, a substance $P$ antagonist (SPA), all resulted in a reduction in the inflammatory response, these were not significant except for capsaicin pretreatment (CAP). ${ }^{*} p<0 \cdot 05$ : differs significantly from control response.

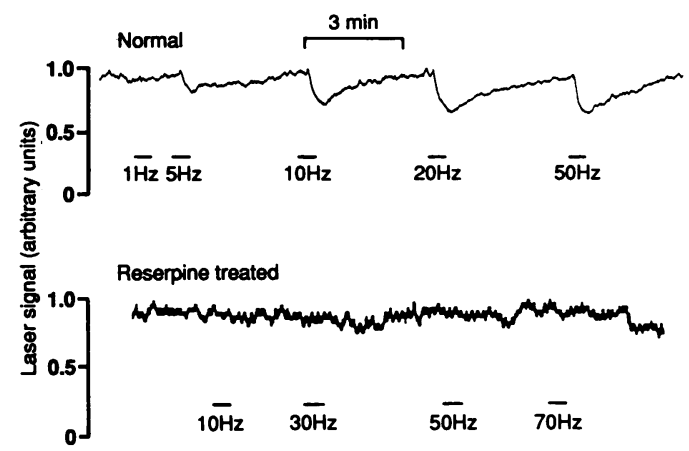

Figure 3 Responses to electrical stimulation $(10 \mathrm{~V} ; 1 \mathrm{~ms}$ width) of the nerve supply to the knee in a normal rat (upper trace) and in a reserpine pretreated rat (lower trace) at different frequencies. Black bars denote periods of stimulation.

In the normal rat electrical stimulation of the nerve supply to the knee ( $10 \mathrm{~V}, 1 \mathrm{~ms}$ width) was effective in reducing articular blood flow with a threshold frequency of $2 \mathrm{~Hz}$ and maximal reduction at $30 \mathrm{~Hz}$, as illustrated for one animal in fig 3 (upper trace). In five out of six reserpine treated rats no vasoconstrictor response to articular nerve stimulation was seen (fig 3, lower trace). The only one of this group of rats which responded to nerve stimulation showed a much smaller maximum vasoconstriction and a higher stimulation threshold $(10 \mathrm{~Hz})$ than shown by normal animals.

\section{Discussion}

Capsaicin is known to produce lasting depletion of unmyelinated afferent fibres when applied topically to peripheral nerves. ${ }^{24}$ The action of a drug is expected to be attenuated after capsaicin treatment if its action is dependent upon the integrity of the unmyelinated afferent fibres supplying the tissue. Surgical removal of the nerves innervating that particular tissue should also result in a similar attenuation on the response. In these studies carrageenan induced inflammation of the rat knee joint was found to follow this expected pattern of action, which is also in accord with our previous findings using carrageenan over a shorter period. ${ }^{21}$

Parratt and West have shown that reserpine treatment is a simple and effective method for depleting sympathetic nerve endings of their neurotransmitters. ${ }^{25}$ Our studies confirmed this view as five of the six reserpine treated rats tested with laser Doppler flowmetry showed no vasoconstrictor response to articular nerve stimulation. The lack of effect of reserpine pretreatment in inhibiting some of the inflammatory responses therefore cannot be ascribed to failure of depletion of neurotransmitters from sympathetic nerve endings. Carrageenan induced inflammation of the rat knee was not affected by reserpine treatment. This suggests that the sympathetic nervous system may not be involved in the carrageenan model of inflammation.

The dose of substance $P$ injected into the synovial cavity $(20 \mu \mathrm{g})$ was chosen to give an inflammatory response comparable with that elicited by the other agents, and cannot be 
considered physiological. This was dispersed around the joint cavity, however, and the actual concentration at the effector sites cannot be determined. Assessments as to the physiological dosage of administered agents are difficult to make as the effectiveness of the agent depends on the tissue concentration achieved at the target tissue and this may be dependent on the point of delivery of the agent. In the case of substance $P$ its release from $C$ fibres may occur in close proximity to its target cells (mast cells and blood vessels), thereby achieving high concentrations locally. Under these circumstances, to obtain comparable concentrations at these sites by intra-articular administration would require a higher concentration of administered substance $P$.

It was of interest to find that inflammation induced by substance $P$ and uric acid were both reduced after capsaicin treatment but were not affected by denervation of the knee. The period of denervation was checked to be sufficient for substantial degeneration of the nerves to occur, as judged by their electron microscopic appearance. The inhibitory effects of capsaicin on substance $P$ and uric acid induced inflammation cannot be attributed to its neurotoxic effects as complete denervation of the knee was ineffective. Possibly capsaicin may have direct inhibitory actions on the target cells which substance $P$ acts upon. ${ }^{22}$ The present findings suggest that capsaicin may also be inhibiting the target cells that uric acid acts upon.

Chemical sympathectomy by reserpine pretreatment showed a significant reduction of the substance $P$ induced inflammation. This is unlikely to be due simply to the depletion of sympathetic neurotransmitters by reserpine, however, as complete surgical denervation of the knee failed to influence the substance $P$ induced response. One of the actions of substance $P$ is to promote mast cell degranulation, ${ }^{26}$ but the possibility of reserpine interfering with this process is also unlikely, as the present studies have shown that the actions of a specific mast cell degranulator, compound $48 / 80$, was not affected by reserpine pretreatment. It is therefore more likely that reserpine is acting through inhibitory actions on other mediators of the substance $P$ response and perhaps on the target cells that substance $P$ affects. In addition to promoting mast cell degranulation, substance $\mathbf{P}$ also causes inflammatory cell chemotaxis, ${ }^{27}$ neutrophil activation, ${ }^{28}$ and fibroblast proliferation, ${ }^{29}$ all of which are recognised components of the arthritic inflammatory process. Substance $P$ has also been shown to activate synoviocytes to secrete prostaglandin $E_{2}$ and collagenase, ${ }^{30}$ as well as to stimulate secretion of interleukin-1like activity from macrophages. ${ }^{31}$ It is therefore possible that reserpine pretreatment inhibits one or more of these processes and thus reduces the substance $\mathbf{P}$ mediated inflammatory response.

In an inverse manner to that of the substance $P$ and uric acid induced inflammation, formalin induced inflammation was not affected by capsaicin pretreatment, but was significantly reduced by surgical denervation. Denervation of the knee could not have altered the mast cell contents or their numbers in the knee joint as the response to compound $48 / 80$ was found to be similar in intact and denervated knees. Inhibition of formalin induced inflammation after denervation is therefore more appropriately explained by the lack of nerve innervation in the knee joint. The failure of capsaicin to attenuate the inflammatory response highlights the fact that this treatment is probably not exertung a neurotoxic effect on articular nerve fibres with the intra-articular mode of administration but may instead act on subsets of cells mediating the inflammatory responses. Hence although formalin induced inflammation is dependent on the nerve supply to the knee, it is not significantly affected by capsaicin pretreatment. The sympathetic nervous system does not seem to play a part in the formalin induced inflammation as no significant alteration of the formalin response was noted after reserpine treatment.

Compound 48/80 induced inflammation was reduced after capsaicin treatment. Direct inhibition of the action of compound $48 / 80$ on mast cells by capsaicin is unlikely as capsaicin was given at least nine days before compound $48 / 80$ injection. It is more likely that the mast cell contents or numbers were reduced as a result of capsaicin administration. The effect of compound $48 / 80$ is also independent of the substance $P$ content in the rat knee as the response to compound $48 / 80$ was the same in either the absence or presence of the substance $P$ antagonist.

We initially concluded that capsaicin treatment and surgical denervation were equivalent procedures and thus gave rise to similar inhibition of the carrageenan induced inflammatory response. ${ }^{21}$ Clearly, however, although this may be true in some models of inflammation, it does not hold in others. Although capsaicin may exert neurotoxic effects, this is not always the sole explanation of its inhibitory actions in the different models of acute inflammation. The substance $P$ and uric acid inflammatory models do not seem to have a neurogenic component as they were unaffected by nerve transection, whereas the presence of intact innervation is important for carrageenan and formalin to exert their full inflammatory actions. Of those inflammatory models which have a neurogenic component, our findings suggest that it is the unmyelinated $\mathrm{C}$ fibres rather than the sympathetic efferent fibres that mediate, at least in part, these inflammatory processes.

Financial support from the Arthritis and Rheumatism Council and ICI Pharmaceuticals is gratefully acknowledged.

1 Bayliss W M. On the origin from the spinal cord of the vasodilator fibres of the hindlimb and on the nature of these fibres. F Physiol (Lond) 1901; 26: 173-209.

2 Jancso N, Jancso-Gabor A, Szolcsanj J. Direct evidence fo neurogenic inflammation and its prevention by denervation and pretreatment with capsaicin. Br $\mathcal{F}$ Pharmacol 1967; 31 : 138-51.

3 Lewis T. The blood vessels of human skin and their responses. London: Shaw, 1927.

4 Lewis T. Experiments relating to cutaneous hyperalgesia and its spread through somatic nerves. Clin Sci 1936; 2: 373-417.

5 Lewis T. Pain. New York: Macmillan, 1941.

6 Gasser H. Unmedullated fibres originating in dorsal root ganglia. F Gen Physiol 1950; 33: 651-90.

Ferrell W R, Russell N J W. Extravasation in the knee induced by antidromic stimulation of articular $\mathrm{C}$ fibre 
afferents of the anaesthetised cat. 7 Physiol (Lond) 1986; 379: 407-16.

8 Ferrell W R, Cant R. Vasodilation of articular blood vessels induced by antidromic electrical stimulation of joint $\mathrm{C}$ fibres. In: Schmidt R F, Schaible H G, Vahle-Hinz C, eds. Fine afferent nerve fibres and pain. Weinheim, FRG: VCH Verlagsgesellschaft, 1987: 187-92.

9 Kenins P. Identification of the unmyelinated sensory nerves which evoke plasma extravasation in response to antidromic which evoke plasma extravasation in response

10 Lembeck F, Holzer $P$. Substance $P$ as neurogenic mediator of antidromic vasodilatation and neurogenic plasma extraantidromic vasodilatation and neurogenic plasma extravasation.

11 Gamse $R$, Holzer P, Lembeck F. Decrease of substance $P$ in primary afferent neurones and impairment of neurogenic plasma extravasation by capsaicin. Br $\mathcal{F}$ Pharmacol 1980 ; 68: 207-13

12 Foreman J C. Peptides and neurogenic inflammation. Br Med Bull 1987; 43: 386-400.

13 Yaksh T L, Baily J, Roddy D R, Harty G J. Peripheral release of substance $P$ from primary afferents. In: Dubner release of substance $P$ from primary afferents. In: Dubner Congress on Pain. 1988: $51-4$.

14 Linde B, Chisolm G, Rosell S. The influence of sympathetic activity and histamine on the blood-tissue exchange of solutes in canine adipose tssue. Acta Physiol Scand 1974; 92: $145-55$.

15 Engel $D$. The influence of sympathetic nervous system on capillary permeability. $\mathcal{F}$ Physiol (Lond) 1941; 99: 161-81.

16 Engel $D$. The influence of the sympathetic nervous system on capillary permeability. Res $E x p M e d(B e r l) 1978 ; 173: 1-8$.

17 Helme R D, Andrews P V. The effects of nerve lesions on the inflammatory response to injury. F Neurosci Res 1985; 13: 453-9.

18 Gozsy B, Kato L. Role of norepinephrine and 5-hydroxytryptamine in the delayed phase of the inflammatory reaction
$553-60$.

19 Green K L. Role of endogenous catecholamines in the anti- intlammatory activity of $\alpha$-adrenoceptor blocking agents. Br $\mathcal{Y}$ Pharmacol 1974; 51: 45-53.

20 Levine J D, Moskowitz M A, Basbaum A I. The contribution of neurogenic inflammation in experimental arthritis. $\mathcal{f}$ Immunol 1985; 135: 843s-7s.

21 Ferrell $\mathbf{W}$ R, Lam F Y. Inhibition of carrageenan induced inflammation in the rat knee joint by substance $P$ antagonist. inflammation in the rat knee joint by

22 Ferrell W R, Lam F Y. Capsaicin suppresses substance Pinduced joint inflammation in the rat. Neurosci Lett 1989; induced joint $155-8$.

23 Harada M, Takeuchi M, Fukao T, Katagiri K. A simple method for the quantitative extraction of dye from skin. $\mathcal{J}$ Pharm Pharmacol 1971; 23: 218-9.

24 Lynn B, Pini A. Long-term block of afferent C-fibres following capsaicin treatment in the rat. $\mathcal{f}$ Physiol (Lond) 1985; 362: 19P.

25 Parratt J R, West G B. Release of 5-hydroxytryptamine and histamine from tissues of the rat. $\mathcal{F}$ Physiol (Lond) 1957; 137: 179-92.

26 Mazurek N, Pecht I, Teichburg V I, Blumberg S. The role of the N-terminal tetrapeptide in the histamine-releasing the N-terminal tetrapeptide in the histamine-releasing

27 Marasco W A, Showell H J, Becker E L. Substance P binds to the formyl peptide chemotaxis receptor on the rabbit neutrophil. Biochem Biophys Res Commun 1981; 99: 1065-72.

28 Bar-Shavit Z, Goldman R, Stabinsky Y, et al. Enhancement of phagocytosis - a newly found activity of substance $P$ residing in its $\mathrm{N}$-terminal tetrapeptide sequence. Biochem Biophys Res Comomun 1980; 94: 1445-51.

29 Nilsson J, von Euler A M, Dalgard C J. Stimulation of connective tissue cell growth by substance $P$ and substance K. Nature 1985; 315: 61-3.

30 Lotz M, Carson D A, Vaughan J H. Substance P activation of rheumatoid synoviocytes: neural pathway in pathogenesis of arthritis. Science 1987; 235: 893-6.

31 Kimball E S, Perisco F'J, Vaughan J H. Substance P, neurokinin A and neurokinin B induce generation of IL-1like activity in P388D1 cells. F Immunol 1988; 141: 3564-9. 